OPEN ACCESS

Edited by:

Pietro Spataro,

Mercatorum University, Italy

Reviewed by:

Serena Mastroberardino,

Sapienza University of Rome, Italy Efrat Barel,

Max Stern Academic College of Emek Yezreel, Israel

*Correspondence:

Martin Asperholm martin.asperholm@ki.se

Specialty section:

This article was submitted to

Cognition,

a section of the journal

Frontiers in Psychology

Received: 20 November 2019

Accepted: 16 March 2020

Published: 17 April 2020

Citation:

Asperholm $M$, van Leuven $L$ and Herlitz A (2020) Sex Differences in

Episodic Memory Variance.

Front. Psychol. 11:613.

doi: 10.3389/fpsyg.2020.00613

\section{Sex Differences in Episodic Memory Variance}

\author{
Martin Asperholm*, Livia van Leuven and Agneta Herlitz \\ Department of Clinical Neuroscience, Karolinska Institutet, Stockholm, Sweden
}

Men as a group have been shown to have larger variances than women in several areas pertaining to both biological and psychological traits, but no investigation has been performed in regard to episodic memory. We conducted an analysis on sex differences in episodic memory variance on 535 studies, representing 962,946 individuals, conducted between 1973 and 2013. Results showed that men had larger variances than women in verbal episodic memory tasks as well as episodic memory tasks having to do with spatial locations. Women, on the other hand, had larger variance than men for tasks involving remembering routes. These effects were for the most part small, and exploratory analyses suggest that they might come about, at least in part, because of measures not sufficiently controlled for ceiling effects. This means that the effects should be interpreted with caution and that further research on sex differences in episodic memory variance is needed.

\section{Keywords: episodic memory, variance, sex differences, mean difference, ceiling effect, meta-analysis}

\section{INTRODUCTION}

With regard to purely physical traits, it can often be shown that men and women differ largely when comparing their respective group averages. One of the most notable examples of a large difference is height where, for example, the average U.S. man is approximately 14 centimeters taller than the average U.S. woman (Fryar et al., 2016). A plethora of differences in how the average man behaves compared to the average woman have also been demonstrated when it comes to many psychological traits, such as aggression (Bettencourt and Miller, 1996), sexual behavior (Petersen and Hyde, 2010), and narcissism (Grijalva et al., 2015). Differences in mean performance between men and women have also been shown for several cognitive domains, such as mathematics, verbal skills (Maylor et al., 2007; Stoet and Geary, 2013, 2015, 2018), and spatial ability (Voyer et al., 1995).

Another such cognitive capability where sex differences in means have been shown is episodic memory, a type of memory concerning the remembrance of the what, where and when of past events that are not contained in working memory (Tulving, 2002). An example of an episodic memory would be to remember what one did yesterday, recalling the content of different events and place them in relation to one another, both in space and time. As such, episodic memory is a multifaceted concept that can be approximated in a number of ways, using different types of material to remember, for example words, images, routes, and faces. However, the most common way to assess episodic memory is by presenting a list of words, and then ask the participants to recall or recognize the earlier presented words. Asperholm et al. (2019a) showed in a large metaanalysis encompassing 617 studies, conducted between 1973 and 2013, that women, as a group, tend to outperform men on episodic memory tasks. However, the material to be remembered affected the magnitude of this advantage, with a female advantage for more verbal tasks, such as words, sentences, and prose, but also for nameable images, and locations. In contrast, there was a male 
advantage in more spatial tasks, such as abstract images and remembering a route. Results from this meta-analysis also indicated that the magnitude of these differences had remained stable since 1973 and that, for verbal tasks, the sex difference was somewhat smaller in childhood and old age than for other ages. Although the underlying mechanism for these sex differences are poorly understood, they have been reported in most of the examined countries (Bonsang et al., 2017; Asperholm et al., 2019b).

Sex differences are, as in the examples above, most often researched and expressed in terms of how the averages of the two sexes compare to each other. However, a somewhat overlooked aspect is to compare how the two sexes vary around the mean. This is an important aspect to consider when investigating sex differences, since it, together with the difference in means, helps to predict the ratio of men and women in the extremes. For example, a large-scale study of IQ scores among Scottish school children showed no difference between the average boy and the average girl (Deary et al., 2003). This could lead one to surmise that there also should be about as many high and low IQ boys as there are high and low IQ girls. However, the variance for boys is larger than for girls (Deary et al., 2003), thereby making boys over-represented at both extremes. On the other hand, asymmetric extremes can often also be observed in cases where there are mean sex differences present. For example, it can be seen that boys are over-represented among the top achievers in mathematical reasoning while girls have similar over-representation in verbal reasoning and writing ability, patterns that come about because of the male advantage in mathematics and the female advantage in verbal abilities (Wai et al., 2010). Thus, simply observing that there are differences in the male to female ratio in the extremes does not automatically reveal what underlying factors contribute to this. Variance is therefore an essential component to investigate in order to understand the bigger picture when it comes to sex differences.

In general, men seem to show larger variability than women. This holds true for physical traits, such as birth weight, blood parameters, and juvenile physical performance (Lehre et al., 2009). Recent pre-published data also indicate a greater male than female variance for subcortical volumes, cortical surface areas, and cortical thickness across the lifespan (Wierenga et al., 2020). The same pattern has also been shown for many cognitive abilities (Feingold, 1992b), such as verbal-, quantitative-, and figural reasoning (Lakin, 2013), as well as for regular school subjects such as mathematics, reading, and science (Hedges and Nowell, 1995; Nowell and Hedges, 1998; Machin and Pekkarinen, 2008). There are, however, also counter-examples. For example, for some progressive matrices tasks, women seem to have larger variances than men (Irwing and Lynn, 2005). Another example comes from a large population-based Romania sample, in which no consistent variance differences between men and women either on general intelligence or second-level specific, cognitive abilities were evident (Iliescu et al., 2016).

To our knowledge, only one previous study has investigated sex differences in episodic memory variance. Hedges and Nowell (1995) computed VR values (ratios of male variance to female variance) on associative memory data, a form of episodic memory, from three national U.S. surveys consisting of over 100,000 high school children. Results were inconclusive with one survey indicating larger variance for males, another larger variance for females, and a third one showing equal variances for males and females.

Why would there be sex differences in variance? One theory attempting to explain why the variance often is found to be larger for men than for women relates it to the difference in sex chromosomes (Reinhold and Engqvist, 2013). In humans, women are the homogametic sex since they have two of the same type of sex chromosome (XX) while men are the heterogametic sex because they have two types of sex chromosomes (XY). The theory states that since genetic expression is a result of the combination of genes on both chromosomes in a chromosome pair, having just a single $\mathrm{X}$ chromosome results in that many of the genes on it cannot be countered by corresponding genes on the less information-rich Y chromosome. This, in turn, means that mutations of a certain gene on the $\mathrm{X}$ chromosome for the heterogametic sex more often will be expressed, resulting in larger variance of expressions of that gene for that group compared with the homogametic sex. In humans, males happen to be the heterogametic sex, but there are also species where females are heterogametic. When comparing variance in body size, it can be shown that it is larger for the heterogametic sex irrespectively of whether that sex is male or female (Reinhold and Engqvist, 2013), in turn showing that the effect can be decoupled from the biological sex. Another theory attempting to explain why men might be more variable than women states that it is due to postnatal factors (Lehre et al., 2009). Here, it is hypothesized that men, because of their slower development, for a longer period of time are exposed to genetic expressions going in different directions. It is also theorized that their ostensibly higher sensitivity to early environmental stressors and opportunities makes them more diverse.

It is unclear to what extent any of the theories outlined above could influence sex differences in variance when it comes to human cognition, and more specifically, episodic memory. In regard to the sex chromosome theory, when examining general intelligence, genes that together are implicated for its expression are over-represented on the $\mathrm{X}$ chromosome compared to the $\mathrm{Y}$ chromosome (Zechner et al., 2001). Hence, the larger variance in males as compared to females in some cognitive abilities, including general intelligence, are at least in line with what would be hypothesized from the chromosome theory (Zechner et al., 2001; Johnson et al., 2009). However, conclusive proof in support of this theory is still missing (Giummo and Johnson, 2012; Printzlau et al., 2017).

As already noted, sex differences in means for episodic memory have been investigated in a large meta-analysis including 617 studies (Asperholm et al., 2019a), but no similar investigation has been conducted on possible differences in variances between the sexes. In order to get a fuller picture of sex differences in episodic memory, we will undertake a large-scale investigation on this topic, using the same dataset as was used in Asperholm et al. (2019a). This investigation will not only determine whether larger male than female variances exist in episodic memory, but it will also either strengthen or weaken the hypothesis 
that men exhibit larger variances in general as compared to women.

Since Asperholm et al. (2019a) could show that the mean sex difference in episodic memory heavily depended on the type of material to be remembered, a similar partition of the data will be analyzed here. That is, the data will be partitioned based on to what degree the task requires verbal (e.g., remembering a word list) or spatial (e.g., remembering a route) processing, in addition to categories that cannot be classified in this way, such as remembering faces or non-visual content. In addition, moderators pertaining to possible bias issues in the dataset (e.g., studies focusing on the topic of sex differences or not) will be investigated as well as age of the participants and year of publication.

In line with the evidence presented above (Feingold, 1992b; Deary et al., 2003; Lehre et al., 2009; Lakin, 2013; Reinhold and Engqvist, 2013), the overall hypothesis is that men will show more variance in episodic memory performance than women for the full dataset as well as for all material categories examined. Although the investigation performed by Hedges and Nowell (1995) on episodic memory reported conflicting results regarding sex differences in variance, there is not enough data to warrant a hypothesis going against the general pattern that has been found. Likewise, based on previous literature, there is no reason to expect that age or publication year should affect the results.

\section{MATERIALS AND METHODS}

\subsection{Dataset}

The data that was used in this study has already been the basis for a meta-analysis on mean sex differences in episodic memory (Asperholm et al., 2019a). All data points in the dataset had to fulfil the following criteria with respect to the study, sample, or performance metric used: (1) the study had to be written in English, published in a peer-reviewed journal, and contain original, empirical data; (2) the sample had to consist of both males and females who had not been selected based on any disorder, disease, or diagnosis and who had not been manipulated in any way that may have affected their normal episodic memory performance; (3) the performance metric had to be based on a controlled and uniform task that was the same for all participants; (4) the performance metric had to assess episodic memory performance.

Potential articles to include were located by querying PsychINFO, PubMed, and Medline with the search terms "memory," "humans," and "sex or gender," limiting the search to articles published from January 1972 to November 2013 (search queries and abstract reading sessions were conducted at two separate occasions, one starting in 2001 and one starting in 2013). This resulted in 9,811 abstracts that, after investigation, were reduced to 3,331 full-text articles that were examined. Out of these, 351 articles were included directly. If the data for a specific article was taken from a large, open database, we opted to get the data directly from the original source instead. Further, author's of 1,047 papers published after 2003 were contacted since it was judged that they might have relevant data not included in the study. This resulted in us acquiring 20 references for new articles where the data in question already were published, and receiving the necessary additional, unpublished data for 246 articles directly from the authors. All in all, this resulted in 617 studies. However, after excluding those studies which lacked variance metrics for men and women, 535 out of these 617 studies remained (see Supplementary Material for a list of these articles), representing 962,946 participants (out of which 56\% were women), spanning in age range from infants to the oldest subjects being 108 years old (see Figure 1 for an overview of the data collection).

\subsection{Moderators}

A number of moderator variables pertaining to the study, sample, and task were recorded and subsequently analyzed. For task specific moderators, data points were categorized into nine categories based on the type of material to be remembered in the episodic memory task, ranging from highly verbal to highly spatial in cases where applicable. These categories were: Verbalwords, sentences, facts, conversations, or narrative content; Images - images of real or abstract objects and scenes; Moviesmovie clips with or without sound; Locations-locations of objects; Routes - routes through space; Faces_images of human faces; Sensory-odors, tastes, and colors; Remaining-material that could not be placed within one of the above categories, such as composite measures based on several of them. Further, each sample was assigned an age value, which was defined as the middle value of the age range whenever mean age was not available. Out of the 535 studies, 467 had data making an age value possible to compute, bringing the mean of all sample ages to 48.1 years $(s d=26.9)$. Year comprised information regarding the year of publication.

In addition, several variables pertaining to possible bias in the dataset were defined. Database search indicates whether the study comes from the first (1972-2001) or second (2001-2013) database query. Data source indicates whether the data was retrieved directly from a published article or whether it was sent to us by authors. Study objective indicates whether it was explicitly stated in the article that one of the research questions was to investigate sex differences. When this was the case, the research question was almost always about difference in means, not about difference in variances. Sampling of subjects indicated whether the sample could be considered a population-based or convenience-based sample. A sample was considered populationbased if it was indicated that the sample was randomly selected from the population.

\subsection{Statistics}

In this study, Hedges' $g$ (Hedges, 1981) and the natural logarithm of the variance ratio ( $\ln V R$; Nakagawa et al., 2015) are used to describe mean and variance differences between two groups, respectively. Hedge's $g$, which is a common measure to describe differences in means, is computed by taking the difference in means between two groups, divide it by their pooled standard deviation, and then multiply the result by a small correction factor in order to hinder small samples from overstating the final estimate. Here, positive values mean that women outperform men and vice versa. The $\mathrm{R}$ package compute.es (Version $0.2-4$; 


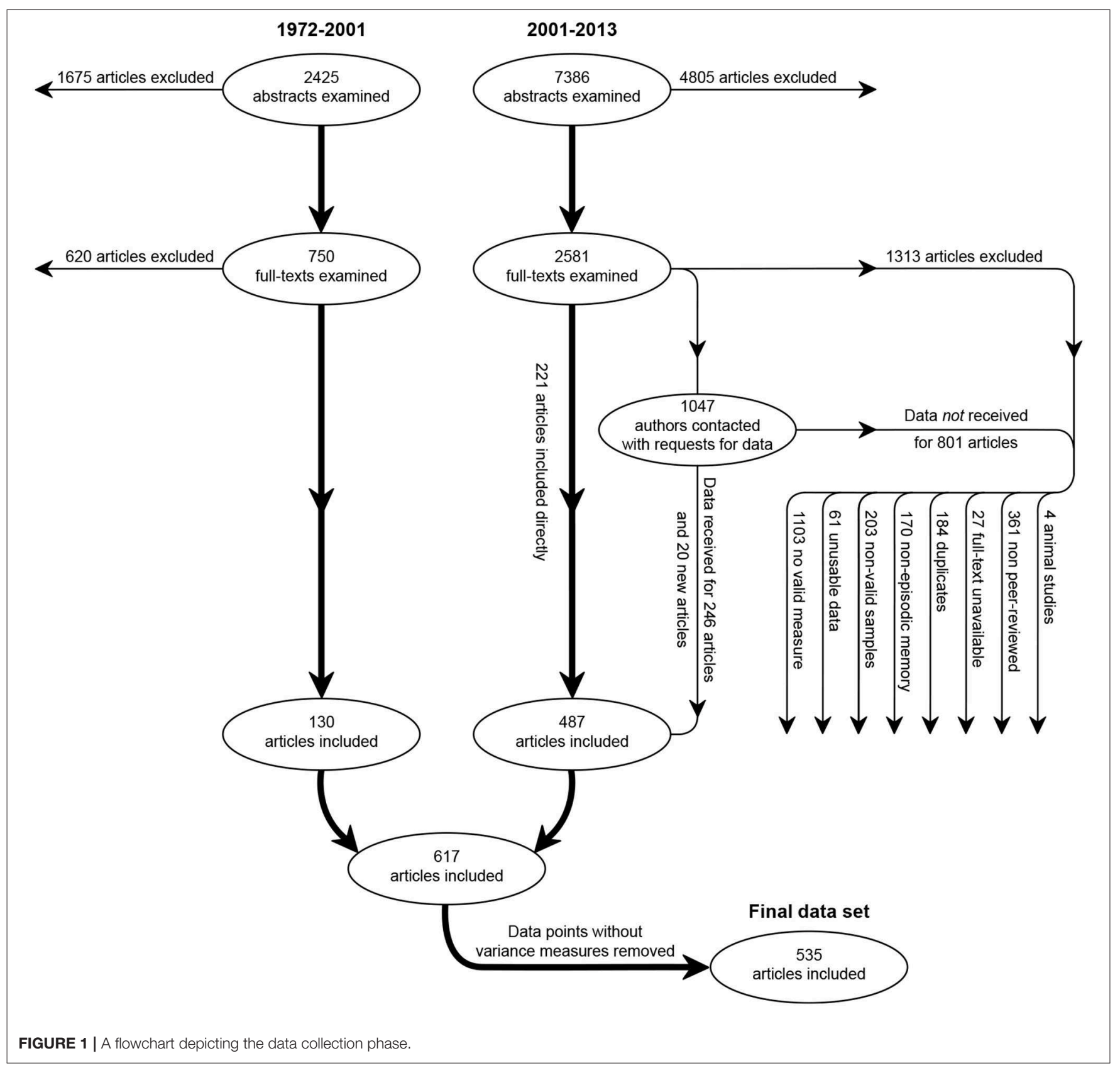

Re, 2013) was used to compute Hedge's $g$ from raw values. $\ln V R$ is basically the natural logarithm of the ratio of two variances. Using just the ratio of two variances $(V R)$ is unproblematic when computing the median variance ratio of several data points, which sometimes is done (Feingold, 1992b; Hedges and Nowell, 1995), but for most analyses and descriptive measures, a measure such as $\ln V R$ is needed in order to avoid giving the variance above the denominator a larger weight than the variance below the denominator in the final measure (Feingold, 1992a). However, $\ln V R$ can always, when analyses have been performed, be converted back to $V R$ for more interpretable results, as is done in this study. Here, Equations (9) and (10) from Nakagawa et al.
(2015) were used to compute $\ln V R$. Positive values mean that men have larger variances than women and vice versa.

Most of the articles in the dataset contributed with several data points. This was because each article could contain several samples (e.g., age groups), where in each of these more than one episodic memory task could be tested (e.g., remembering a number of common words and remembering a number of abstract images), some of them reporting more than one dependent measure (e.g., free or cued recall; immediate or delayed recognition). To account for this hierarchical structure of the data, all analyses performed were five-level randomeffects meta-analyses/meta-regressions, carried out using the 
rma.mv function in the $\mathrm{R}$ package metafor (Viechtbauer, 2010). The alpha level was always set to 0.05 for all individual analyses.

\section{RESULTS}

First, a limited replication of the meta-analysis performed in Asperholm et al. (2019a) was carried out on the somewhat reduced dataset (535 studies instead of 617). This entailed running a meta-analysis with Hedge's $g$ (with positive values indicating that women performed at a higher level than men) as the dependent variable, first without any moderators and then with material category as a moderator (see Table 1). These analyses showed similar results as in Asperholm et al. (2019a), with the categories Movies and Remaining going from being significantly different from zero to being non-significant. Results

TABLE 1 | Estimated effect sizes from a meta-analysis with Hedge's $g$ as the dependent variable and material category as a moderator (Omnibus $p<0.001 ;{ }^{2}$ $=89 \%$ )

\begin{tabular}{lccccc}
\hline Type of material & $\boldsymbol{g}$ & $\mathbf{9 5 \%} \mathbf{C l}$ & $\boldsymbol{k}$ & $\boldsymbol{p}$ & $\boldsymbol{I}^{\mathbf{2}} \mathbf{( \% )}$ \\
\hline Verbal & 0.26 & {$[0.24,0.29]$} & 333 & $<0.001$ & 88 \\
Images & 0.00 & {$[-0.04,0.04]$} & 174 & 0.98 & 85 \\
Movies & 0.10 & {$[-0.03,0.22]$} & 19 & 0.13 & 48 \\
Locations & 0.16 & {$[0.10,0.21]$} & 56 & $<0.001$ & 96 \\
Routes & -0.18 & {$[-0.31,-0.05]$} & 15 & 0.01 & 70 \\
Faces & 0.23 & {$[0.15,0.30]$} & 47 & $<0.001$ & 74 \\
Sensory & 0.32 & {$[0.12,0.51]$} & 7 & 0.00 & 57 \\
Remaining & 0.07 & {$[-0.00,0.13]$} & 60 & 0.053 & 68 \\
Total & 0.17 & {$[0.15,0.20]$} & 535 & $<0.001$ & 89 \\
\hline
\end{tabular}

Each row indicates whether the effect size of that specific level of the material category moderator is reliably different from 0 . Estimate for Total is based on a meta-analysis using no moderators. $g=$ Hedge's $\mathrm{g}$; $95 \% \mathrm{Cl}=$ the $95 \%$ confidence interval of the estimate; $k$ = number of studies; $p=$ the $p$-value; $R^{2}=$ statistics denoting the percentage of variation across studies that is due to heterogeneity rather than due to chance. showed that women outperformed men on the categories Verbal, Faces, Locations, and Sensory, while men outperformed women on the category Routes.

After this, the same analyses as outlined above but with $\ln V R$ (with positive values indicating that men had larger variance than women) as the dependent variable were performed (see Figure 2). For the overall analysis using no moderators, the result was significantly different from zero, with men having larger variance than women. For the moderator analysis, the estimated effect sizes for the categories Verbal, Locations (where males had larger variance than females), and Routes (where females had larger variance than males) were significantly different from zero.

Next, separate meta-regressions on all material category subsets, including the Total dataset, were performed using age (see Table 2) and year of publication (see Table 3 ) as moderators. For year, no linear relationships could be found for any of the different subsets, and for age only the analyses for the Verbal and Total subsets showed significant, small, negative linear relationships, indicating that the men's larger variance became smaller across age.

In order to determine potential biases in the dataset, four moderator analyses were conducted with $\ln V R$ as the dependent variable (see Table 4): First, we compared effect sizes retrieved from the first database search (1972-2001) with those from the second (2001-2013). Second, we compared effect sizes that were retrieved directly from publications with those that were sent to us from the authors. Third, we compared effect sizes from studies where the objective in the study was to investigate sex differences and effect sizes from studies where no such objective was present. Fourth, we compared effect sizes based on whether the sampling was population-based or not. This was also the only of the analyses that had a significant omnibus test, showing that the variance sex difference was more positive (i.e., toward men having larger variance) in convenience-based samples compared with population-based samples.

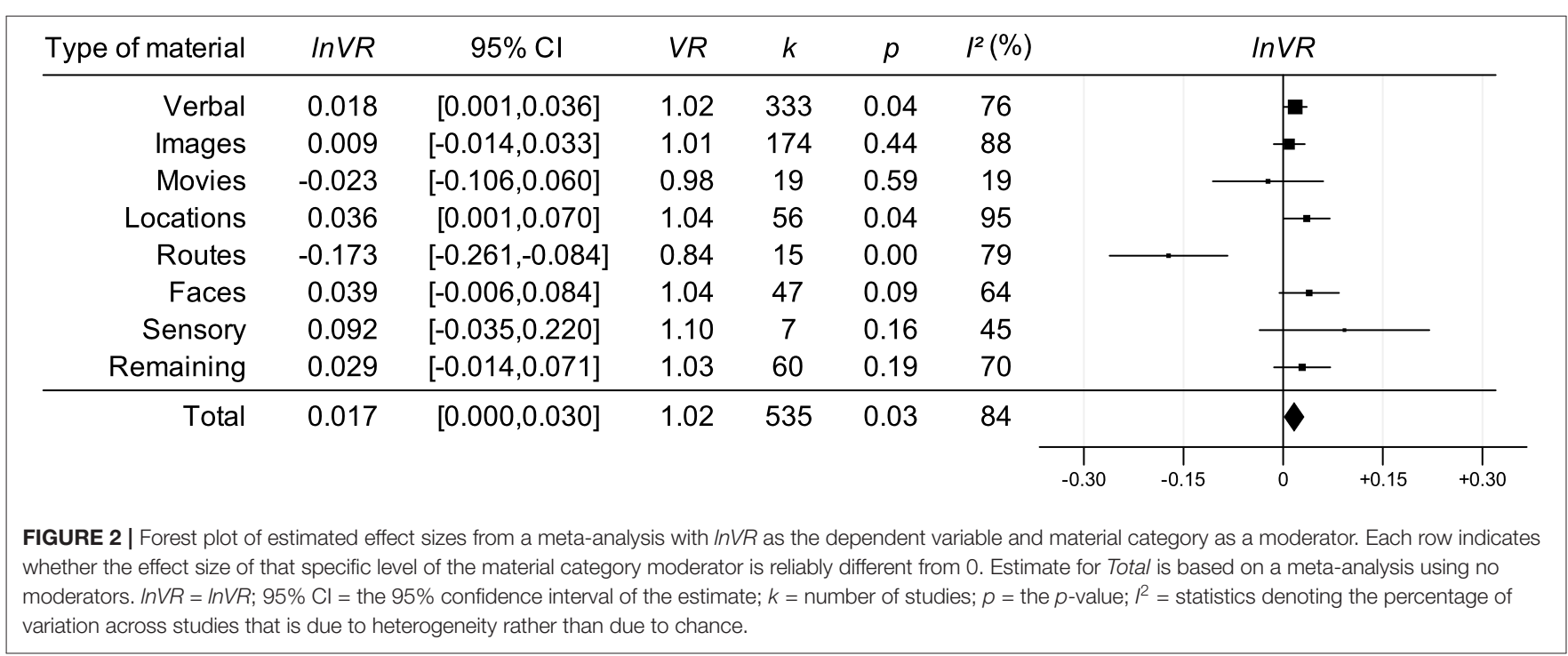


TABLE 2 | Best-fit intercepts and regression lines estimated from separate meta-regressions with InVR as the dependent variable and age as a moderator.

\begin{tabular}{|c|c|c|c|c|c|c|}
\hline Moderator-level & & $\ln V R$ & $95 \% \mathrm{Cl}$ & $k$ & $p$ & $I^{2}(\%$ \\
\hline \multirow[t]{3}{*}{ Verbal } & & & & 296 & & 76 \\
\hline & Intercept & 0.094 & {$[0.067,0.122]$} & & $<0.001$ & \\
\hline & Age & -0.001 & {$[-0.002,-0.001]$} & & $<0.001$ & \\
\hline \multirow[t]{3}{*}{ Images } & & & & 153 & & 89 \\
\hline & Intercept & 0.043 & {$[-0.023,0.116]$} & & 0.25 & \\
\hline & Age & -0.001 & {$[-0.003,0.001]$} & & 0.19 & \\
\hline \multirow[t]{3}{*}{ Movies } & & & & 15 & & 38 \\
\hline & Intercept & 0.028 & {$[-0.133,0.189]$} & & 0.73 & \\
\hline & Age & -0.003 & {$[-0.008,0.003]$} & & 0.35 & \\
\hline \multirow[t]{3}{*}{ Locations } & & & & 48 & & 96 \\
\hline & Intercept & 0.035 & {$[-0.053,0.123]$} & & 0.44 & \\
\hline & Age & -0.001 & {$[-0.003,0.002]$} & & 0.53 & \\
\hline \multirow[t]{3}{*}{ Routes } & & & & 14 & & 78 \\
\hline & Intercept & -0.374 & {$[-0.752,0.004]$} & & 0.053 & \\
\hline & Age & 0.008 & {$[-0.004,0.020]$} & & 0.19 & \\
\hline \multirow[t]{3}{*}{ Faces } & & & & 41 & & 66 \\
\hline & Intercept & 0.025 & {$[-0.089,0.139]$} & & 0.67 & \\
\hline & Age & 0.000 & {$[-0.003,0.004]$} & & 0.82 & \\
\hline \multirow[t]{3}{*}{ Sensory } & & & & 7 & & 58 \\
\hline & Intercept & -0.105 & {$[-0.403,0.194]$} & & 0.49 & \\
\hline & Age & 0.006 & {$[0.000,0.011]$} & & 0.052 & \\
\hline \multirow[t]{3}{*}{ Remaining } & & & & 46 & & 62 \\
\hline & Intercept & -0.006 & {$[-0.102,0.090]$} & & 0.90 & \\
\hline & Age & 0.000 & {$[-0.003,0.003]$} & & 0.87 & \\
\hline \multirow[t]{3}{*}{ Total } & & & & 467 & & 85 \\
\hline & Intercept & 0.055 & {$[0.029,0.081]$} & & $<0.001$ & \\
\hline & Age & -0.001 & {$[-0.001,-0.001]$} & & $<0.001$ & \\
\hline
\end{tabular}

InVR = InVR; $95 \% \mathrm{Cl}=$ the $95 \%$ confidence interval of the estimate; $k=$ number of studies; $p=$ the $p$-value; $R^{2}$ = statistics denoting the percentage of variation across studies that is due to heterogeneity rather than due to chance.

Finally, individual meta-regressions with $\ln V R$ as the dependent variable and Hedge's $g$ as the moderator were performed as exploratory analyses for all categories (see Table 5). Results showed significant, positive relationships for Total, Verbal, Images, Locations, Routes, and Faces, and a significant, negative relationship for Sensory. A positive relationship here indicates that as women perform higher and higher compared to men, their variance also becomes smaller and smaller compared to the men's (and vice versa). An illustration of this effect can be seen in Figure 3 where the association is shown for the Total dataset.

In Figure 4, assumed distributions of male and female performance have been plotted based on the estimates of Hedge's $g$ (see Table 1) and $\ln V R$ (see Figure 2). Also, in Figure 5, a funnel plot for the full dataset is shown, and the result from a meta-regression with $\ln V R$ as the dependent variable and sample size/logged sample size as a moderator is reported, showing no detectable relationship between the two, indicating that the funnel plot is symmetrical.
TABLE 3 | Best-fit intercepts and regression lines estimated from separate meta-regressions with InVR as the dependent variable and publication year of the studies as a moderator.

\begin{tabular}{|c|c|c|c|c|c|c|}
\hline Moderator-level & & $\ln V R$ & $95 \% \mathrm{Cl}$ & $k$ & $p$ & $I^{2}(\%)$ \\
\hline \multirow[t]{3}{*}{ Verbal } & & & & 333 & & 75 \\
\hline & Intercept & -1.248 & {$[-5.625,3.129]$} & & 0.58 & \\
\hline & Year & 0.001 & {$[-0.002,0.003]$} & & 0.57 & \\
\hline \multirow[t]{3}{*}{ Images } & & & & 174 & & 88 \\
\hline & Intercept & 3.140 & {$[-4.821,11.102]$} & & 0.44 & \\
\hline & Year & -0.002 & {$[-0.006,0.002]$} & & 0.44 & \\
\hline \multirow[t]{3}{*}{ Movies } & & & & 19 & & 22 \\
\hline & Intercept & 14.884 & {$[-19.069,48.836]$} & & 0.39 & \\
\hline & Year & -0.007 & {$[-0.024,0.010]$} & & 0.39 & \\
\hline \multirow[t]{3}{*}{ Locations } & & & & 56 & & 95 \\
\hline & Intercept & -7.568 & {$[-19.692,4.557]$} & & 0.22 & \\
\hline & Year & 0.004 & {$[-0.002,0.010]$} & & 0.22 & \\
\hline \multirow[t]{3}{*}{ Routes } & & & & 15 & & 78 \\
\hline & Intercept & -31.090 & {$[-81.667,19.486]$} & & 0.23 & \\
\hline & Year & 0.015 & {$[-0.010,0.041]$} & & 0.23 & \\
\hline \multirow[t]{3}{*}{ Faces } & & & & 47 & & 63 \\
\hline & Intercept & 11.348 & {$[-1.046,23.741]$} & & 0.07 & \\
\hline & Year & -0.006 & {$[-0.012,0.001]$} & & 0.07 & \\
\hline \multirow[t]{3}{*}{ Sensory } & & & & 7 & - & - \\
\hline & Intercept & - & - & & - & \\
\hline & Year & - & - & & - & \\
\hline \multirow[t]{3}{*}{ Remaining } & & & & 60 & & 70 \\
\hline & Intercept & -0.823 & {$[-13.990,12.344]$} & & 0.90 & \\
\hline & Year & 0.000 & {$[-0.006,0.007]$} & & 0.90 & \\
\hline \multirow[t]{3}{*}{ Total } & & & & 535 & & 83 \\
\hline & Intercept & -0.960 & {$[-4.685,2.765]$} & & 0.61 & \\
\hline & Year & 0.001 & {$[-0.001,0.002]$} & & 0.61 & \\
\hline
\end{tabular}

InVR = InVR; $95 \% \mathrm{Cl}=$ the $95 \%$ confidence interval of the estimate; $k=$ number of studies; $p=$ the $p$-value; $R^{2}$ = statistics denoting the percentage of variation across studies that is due to heterogeneity rather than due to chance. Note that the analysis for the Sensory subset did not converge due to too little variance in the year of publication variable.

\section{DISCUSSION}

The goal of this study was to investigate possible sex differences in variance for episodic memory performance, where the hypothesis was that men would show larger variances than women regardless of the material to be remembered and that no variance differences would be seen for age or publication year. This was done by fitting a set of meta-analytical models on a dataset of 535 studies, previously used for a meta-analysis on sex differences in episodic memory mean performance (Asperholm et al., 2019a).

Results showed that there was a sex difference in variance for the full dataset with men having larger variances than women. Further, when running a moderator analysis with type of material to be remembered, men had significantly larger variances in both Verbal and Locations, whereas women had significantly larger variance for Routes (see Figure 2). No sex difference could be detected in the five remaining categories. These results are 
TABLE 4 | Estimated effect sizes from meta-analyses with InVR as the dependent variable and four different moderators pertaining to possible bias.

\begin{tabular}{|c|c|c|c|c|c|c|}
\hline Moderator-level & & $\ln V R$ & $95 \% \mathrm{Cl}$ & $k$ & $p$ & $I^{2}(\%)$ \\
\hline \multirow[t]{3}{*}{ Database search } & & & & 535 & 0.76 & 83 \\
\hline & 1972-2001 & 0.012 & {$[-0.023,0.046]$} & 91 & 0.51 & \\
\hline & 2001-2013 & 0.018 & {$[0.001,0.034]$} & 444 & 0.04 & \\
\hline \multirow[t]{3}{*}{ Data source } & & & & 535 & 0.71 & 84 \\
\hline & $\begin{array}{l}\text { Retrieved from } \\
\text { publications }\end{array}$ & 0.019 & {$[-0.001,0.034]$} & 288 & 0.06 & \\
\hline & $\begin{array}{l}\text { Received from } \\
\text { authors }\end{array}$ & 0.013 & {$[-0.009,0.036]$} & 247 & 0.24 & \\
\hline \multirow[t]{3}{*}{ Study objective } & & & & 535 & 0.14 & 84 \\
\hline & $\begin{array}{l}\text { Sex } \\
\text { differences }\end{array}$ & 0.008 & {$[-0.011,0.026]$} & 204 & 0.42 & \\
\hline & $\begin{array}{l}\text { Other } \\
\text { objectives }\end{array}$ & 0.031 & {$[0.007,0.055]$} & 331 & 0.01 & \\
\hline \multirow{3}{*}{ Sampling of subjects } & & & & 522 & 0.03 & 84 \\
\hline & $\begin{array}{l}\text { Convenience- } \\
\text { based }\end{array}$ & 0.020 & {$[0.004,0.036]$} & 472 & 0.01 & \\
\hline & $\begin{array}{l}\text { Population- } \\
\text { based }\end{array}$ & -0.021 & {$[-0.056,0.015]$} & 53 & 0.25 & \\
\hline
\end{tabular}

Each row indicates whether the effect size of that specific level of the moderator is reliably different from 0 . Results from omnibus tests are presented on the same row as the moderator name. Estimate for Total is based on a meta-analysis using no moderators. InVR = InVR; $95 \% \mathrm{Cl}=$ the $95 \%$ confidence interval of the estimate; $k$ = number of studies; $p=$ the $p$-value; $I^{2}=$ statistics denoting the percentage of variation across studies that is due to heterogeneity rather than due to chance.

somewhat reminiscent of the inconsistency of results that Hedges and Nowell (1995) presented when investigating associative memory, where there also were examples of men being more variable, women being more variable, and no difference at all. However, our results were not in line with the hypothesis that men would be more variable regardless of material to be remembered.

Further, results showed that there were significant linear relationships between age and sex differences in variance for Verbal and Total, indicating that the men's larger variances became smaller across age. Although the reason for this finding is unclear, it should be noted that age for each sample in this analysis was rather unspecific. That is, age was computed by either taking the mean age of the sample or, alternatively, the middle value of the age range. The latter measure can differ quite substantially from the mean age and in both cases, variance around the age value was never taken into account. Further research is needed before concluding that the sex difference in variance become smaller across age in episodic memory.

It is not likely that publication bias can explain the significant sex differences in variance as the same results were found regardless of whether data were taken directly from publications or were previously unreported data obtained from authors. Similarly, whether or not there was an explicit objective of the study to investigate potential sex differences did not impact on the results. Out of the moderator analyses performed that pertained to possible bias in the dataset (see Table 4), including meta-regressions with publication year as moderator
TABLE 5 | Best-fit intercepts and regression lines estimated from separate meta-regressions with InVR as the dependent variable and Hedge's $g$ as a moderator.

\begin{tabular}{|c|c|c|c|c|c|c|}
\hline Moderator-level & & $\ln V R$ & $95 \% \mathrm{Cl}$ & $k$ & $p$ & $I^{2}(\%)$ \\
\hline \multirow[t]{3}{*}{ Verbal } & & & & 333 & & 76 \\
\hline & Intercept & 0.023 & {$[0.006,0.040]$} & & 0.01 & \\
\hline & Hedge's g & 0.032 & {$[0.009,0.055]$} & & 0.01 & \\
\hline \multirow[t]{3}{*}{ Images } & & & & 174 & & 88 \\
\hline & Intercept & 0.006 & {$[-0.029,0.041]$} & & 0.74 & \\
\hline & Hedge's g & 0.143 & {$[0.097,0.190]$} & & $<.001$ & \\
\hline \multirow[t]{3}{*}{ Movies } & & & & 19 & & 19 \\
\hline & Intercept & -0.005 & {$[-0.058,0.048]$} & & 0.86 & \\
\hline & Hedge's g & -0.030 & {$[-0.145,0.085]$} & & 0.61 & \\
\hline \multirow[t]{3}{*}{ Locations } & & & & 56 & & 95 \\
\hline & Intercept & -0.012 & {$[-0.049,0.024]$} & & 0.51 & \\
\hline & Hedge's g & 0.211 & {$[0.121,0.301]$} & & $<0.001$ & \\
\hline \multirow[t]{3}{*}{ Routes } & & & & 15 & & 79 \\
\hline & Intercept & -0.052 & {$[-0.208,0.104]$} & & 0.51 & \\
\hline & Hedge's g & 0.408 & {$[0.199,0.618]$} & & 0.00 & \\
\hline \multirow[t]{3}{*}{ Faces } & & & & 47 & & 64 \\
\hline & Intercept & 0.006 & {$[-0.045,0.058]$} & & 0.81 & \\
\hline & Hedge's g & 0.178 & {$[0.101,0.255]$} & & $<0.001$ & \\
\hline \multirow[t]{3}{*}{ Sensory } & & & & 7 & & 45 \\
\hline & Intercept & 0.167 & {$[-0.015,0.349]$} & & 0.07 & \\
\hline & Hedge's g & -0.128 & {$[-0.216,-0.041]$} & & 0.00 & \\
\hline \multirow[t]{3}{*}{ Remaining } & & & & 60 & & 70 \\
\hline & Intercept & -0.006 & {$[-0.058,0.046]$} & & 0.83 & \\
\hline & Hedge's g & 0.023 & {$[-0.084,0.130]$} & & 0.68 & \\
\hline \multirow[t]{3}{*}{ Total } & & & & 535 & & 84 \\
\hline & Intercept & 0.003 & {$[-0.012,0.018]$} & & 0.67 & \\
\hline & Hedge's g & 0.072 & {$[0.054,0.090]$} & & $<0.001$ & \\
\hline
\end{tabular}

InVR = InVR; $95 \% \mathrm{Cl}=$ the $95 \%$ confidence interval of the estimate; $k=$ number of studies; $p=$ the $p$-value; $R^{2}$ = statistics denoting the percentage of variation across studies that is due to heterogeneity rather than due to chance.

(see Table 3), it was only whether the sample was populationbased or convenience-based that could be shown to have an effect. However, it is not clear why men had larger variance in convenience-based samples than in population-based samples. Again, a replication of this finding would strengthen the results.

For all of the categories where variance differences could be detected, meta-regressions showed that when the mean sex differences became larger, the variances of the excelling sex became smaller (see Table 5 and Figure 3). This could come about because of a ceiling effect, where as one sex approaches the ceiling in a certain task, their variance would also be constricted, which in turn would make their variance smaller compared to the other sex. In cases where a ceiling effect would be the result of deficiencies in how the task was constructed (for example, a word list consisting of fewer words than what some of the participants are capable of remembering), rather than because of an actual performance limit, this would contribute toward overestimating the true, underlying variance difference. 
When considering the estimated $\ln V R$ effect sizes of the material categories (see Figure 2), it can be seen that whenever men had larger variances than women, women also outperformed men, and vice versa. This is in line with the reasoning above, where a ceiling effect would constrict the variance of the higher performing sex more. However, even if this would be the case, it is not clear whether this potential ceiling effect comes about because of methodological or functional reasons, something that would drastically affect

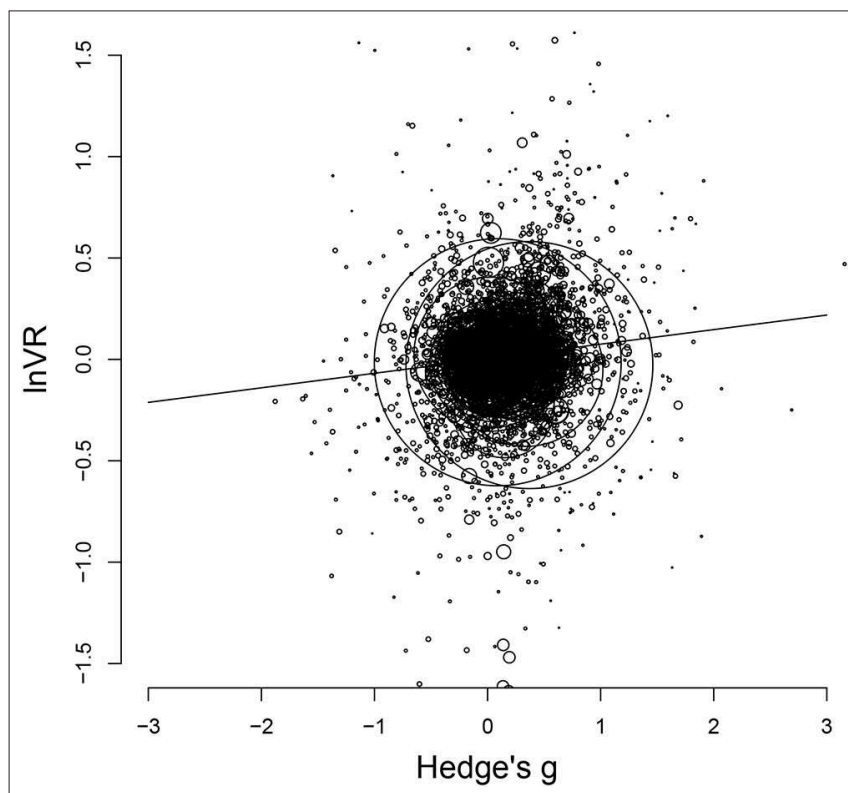

FIGURE 3 | Scatterplot of InVR and Hedge's $g$ for the full dataset. The diameter of each data point is equal to the inverse of its squared variance. The line shows the best-fitting regression (see Table 5). the interpretation of the findings. This reasoning should also be viewed in the context of the reported sex differences in variances for the most part being rather small. For example, for Verbal, which showed the smallest effect $(V R=1.02)$, assuming equal means of the two groups would result in

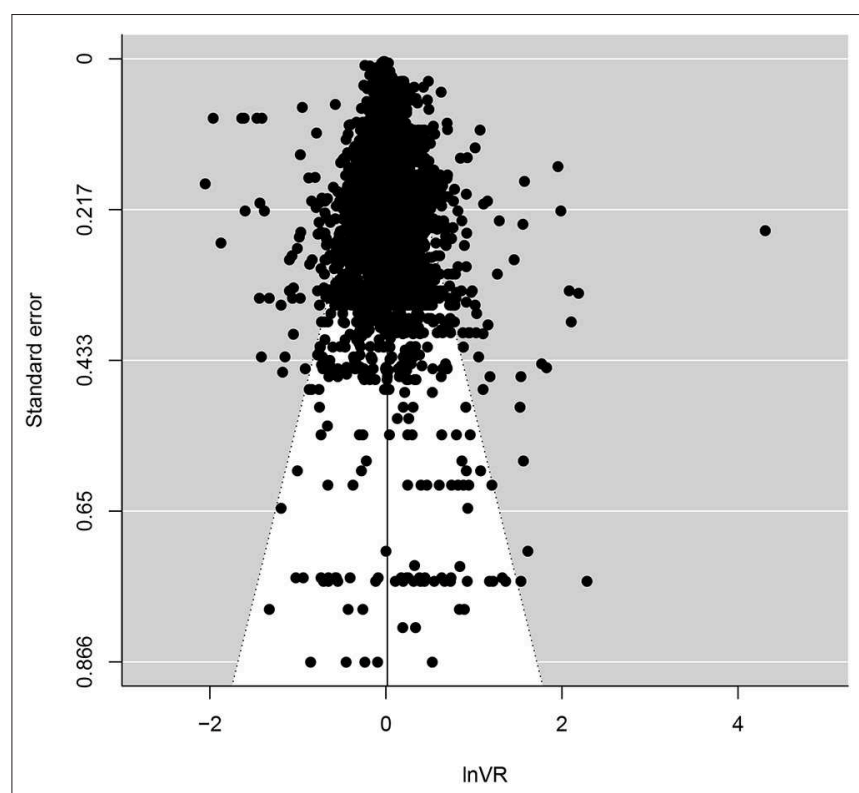

FIGURE 5 | Funnel plot for the full dataset with $\ln V R$ on the $x$-axis and standard error on the $y$-axis. A meta-regression with $\ln V R$ as the dependent variable and sample size as a moderator showed no relationship between the two (Intercept: 0.017 [0.002, 0.032], $p=.02$; Sample size: 0.000 [0.000, $0.000], p=.30$ ). A meta-regression with $\ln V R$ as the dependent variable and logged (with the natural logarithm) sample size as a moderator showed no relationship between the two either (Intercept: 0.026 [-0.017, 0.060], $p=.28$; Logged sample size: -0.001 [-0.008, 0.006], $p=.78$ ).

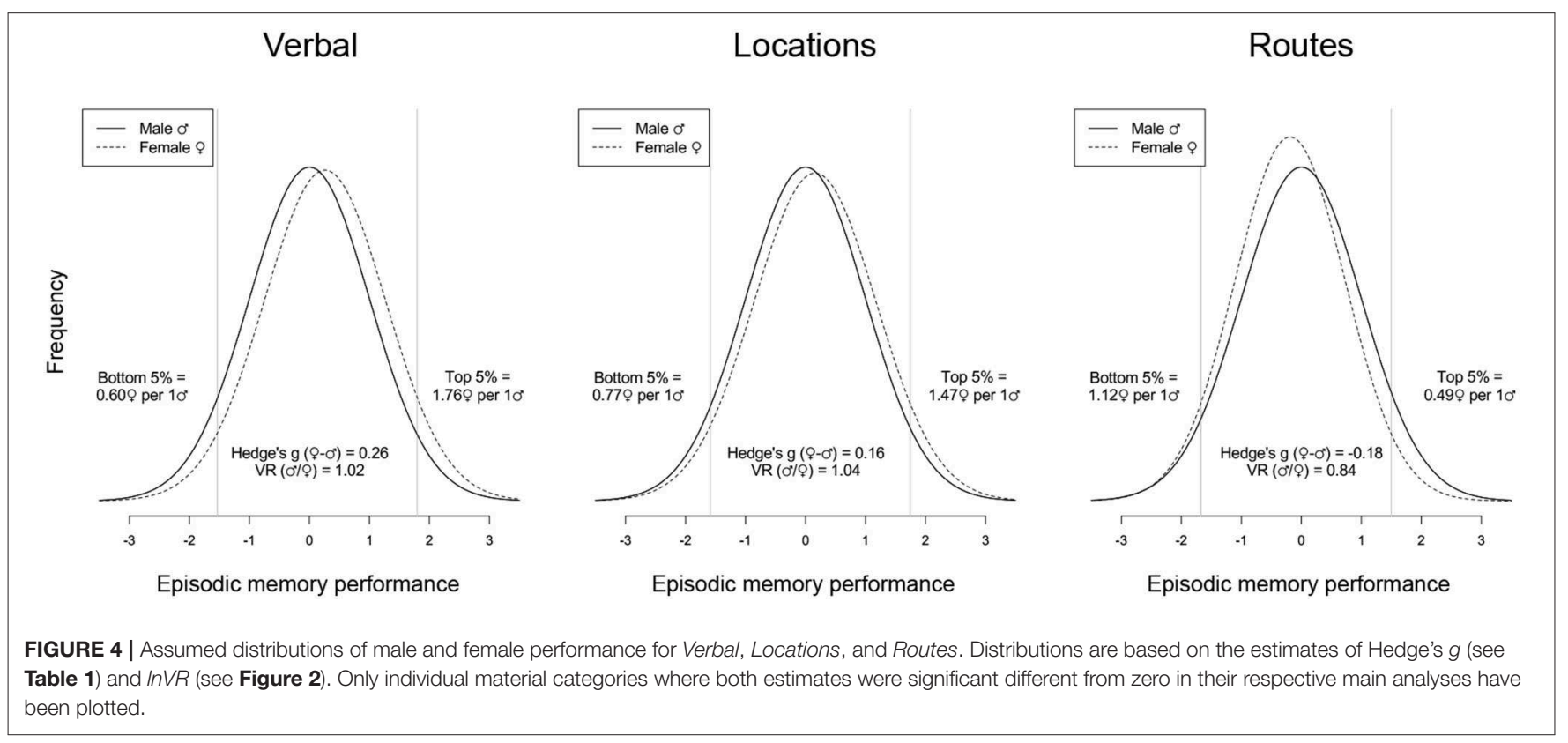


1.03 men for every woman in the top/bottom 5\%. Taken together, the sex differences in variance that were found (see Figure 2) should be interpreted with caution. Also, if ceiling effects indeed are affecting the outcome this would suggest that the actual sex differences in means, if anything, might be underestimated.

Investigating whether a ceiling effect contributes to the effect in other fields where sex differences in variance have been found would require a thorough examination of the underlying data. However, pointing toward a number of examples where one sex have larger variances while also performing at a higher level would at least indicate that a possible ceiling effect can not explain the sex differences in variance in full. Examples of this, when means and variances go in the same direction, include mathematics (Hedges and Nowell, 1995; Nowell and Hedges, 1998; Machin and Pekkarinen, 2008) and quantitative reasoning (Lakin, 2013).

A limitation of the present study is that the analyses were designed to only test for whether there are differences in variances between men and women, meaning that the results presented only can provide evidence for sex differences. Non-significant results only indicate that there is not enough power to detect a difference. Thus, the non-significant results can neither be used to determine that there are no sex differences in variance, nor to determine that differences in variances are small enough to be meaningless.

\section{CONCLUSION}

In summary, some support was found for the hypothesis that men have more variance than women on episodic memory tasks, but only in two out of eighth material categories investigated (Verbal and Locations), and with a reversed effect found in one of the categories (Routes). However, exploratory analyses indicated that these, for the most part very small, effects potentially could be exaggerated because of ceiling effects resulting from limitations in testing procedures. The result should therefore be interpreted with caution. Future research should more thoroughly investigate whether there is a ceiling effect present in fields where men

\section{REFERENCES}

Asperholm, M., Högman, N., Rafi, J., and Herlitz, A. (2019a). What did you do yesterday? Sex differences in episodic memory. Psychol. Bull. 145, 785-821. doi: $10.1037 /$ bul0000197

Asperholm, M., Nagar, S., Dekhtyar, S., and Herlitz, A. (2019b). The magnitude of sex differences in verbal episodic memory increases with social progress: data from 54 countries across 40 years. PLoS ONE 14:e214945. doi: 10.1371/journal.pone.0214945

Bettencourt, B. A., and Miller, N. (1996). Gender differences in aggression as a function of provocation: a meta-analysis. Psychol. Bull. 119, 422-447. doi: 10.1037/0033-2909.119.3.422

Bonsang, E., Skirbekk, V., and Staudinger, U. (2017). As you sow, so shall you reap: gender-role attitudes and late-life cognition. Psychol. Sci. 28:095679761770863. doi: 10.1177/0956797617708634

Deary, I. J., Thorpe, G., Wilson, V., Starr, J. M., and Whalley, L. J. (2003). Population sex differences in iq at age 11: the scottish mental survey 1932. Intelligence 31, 533-542. doi: 10.1016/S0160-2896(03) 00053-9 and women differ in variance, and further, what the nature of this ceiling effect is. The topic of this article is an important question to elucidate since differences in variances also influences the number of men and women found at different levels of performance, even if there is a lack of mean differences between the two groups.

\section{DATA AVAILABILITY STATEMENT}

All datasets generated for this study are included in the article/Supplementary Material.

\section{AUTHOR CONTRIBUTIONS}

$\mathrm{AH}$ came up with the idea for the study. MA designed the research and carried out the analysis. MA and LL wrote the manuscript. All authors read and commented on the research.

\section{ACKNOWLEDGMENTS}

This research was supported by grants awarded to Agneta Herlitz by the Swedish Research Council and FORTE. The large amount of data in this study was made available through work by, foremost, Nadja Högman and Jonas Rafi, but also by Emelie von Vogelsang Antonsson, Hannes Bäckman, Elin Frögéli, Erik Grimsby, Linnea Jesinkey, Maria Larsson, Catharina Lewin, Hillevi Johansson Lignell, Severin Lindskog, Tone Nordling, Christian Plahn, Jenny Rehnman, Markus Söderqvist, and Marielouice Wänseth. We are also grateful to many researcher's generosity and willingness to contribute with important information (see Asperholm et al., 2019a, for a list of these authors).

\section{SUPPLEMENTARY MATERIAL}

The Supplementary Material for this article can be found online at: https://www.frontiersin.org/articles/10.3389/fpsyg. 2020.00613/full\#supplementary-material

Feingold, A. (1992a). Cumulation of variance ratios. Rev. Educ. Res. 62, 433-434. doi: 10.3102/00346543062004433

Feingold, A. (1992b). Sex differences in variability in intellectual abilities: a new look at an old controversy. Rev. Educ. Res. 62, 61-84. doi: 10.3102/00346543062001061

Fryar, C. D., Gu, Q., Ogden, C. L., and Flegal, K. M. (2016). Anthropometric reference data for children and adults: United states, 2011-2014. Vital Health Stat. 3, 1-46.

Giummo, J., and Johnson, W. (2012). Testing for evidence of an x-linked genetic basis for a greater proportion of males with high cognitive ability. Behav. Genet. 42, 808-819. doi: 10.1007/s10519-012-9552-z

Grijalva, E., Newman, D. A., Tay, L., Donnellan, M. B., Harms, P. D., Robins, R. W., et al. (2015). Gender differences in narcissism: a meta-analytic review. Psychol. Bull. 141, 261-310. doi: 10.1037/a0038231

Hedges, L. V. (1981). Distribution theory for glass's estimator of effect size and related estimators. J. Educ. Stat. 6, 107-128. doi: 10.3102/10769986006002107

Hedges, L. V., and Nowell, A. (1995). Sex differences in mental test scores, variability, and numbers of high-scoring individuals. Science 269, 41-45. doi: $10.1126 /$ science.7604277 
Iliescu, D., Ilie, A., Ispas, D., Dobrean, A., and Clinciu, A. I. (2016). Sex differences in intelligence: a multi-measure approach using nationally representative samples from romania. Intelligence 58, 54-61. doi: 10.1016/j.intell.2016.06.007

Irwing, P., and Lynn, R. (2005). Sex differences in means and variability on the progressive matrices in university students: a meta-analysis. Br. J. Psychol. 96, 505-524. doi: 10.1348/000712605X53542

Johnson, W., Carothers, A., and Deary, I. J. (2009). A role for the x chromosome in sex differences in variability in general intelligence? Perspect. Psychol. Sci. 4, 598-611. doi: 10.1111/j.1745-6924.2009.01168.x

Lakin, J. M. (2013). Sex differences in reasoning abilities: surprising evidence that male-female ratios in the tails of the quantitative reasoning distribution have increased. Intelligence 41, 263-274. doi: 10.1016/j.intell.2013.04.004

Lehre, A.-C., Lehre, K. P., Laake, P., and Danbolt, N. C. (2009). Greater intrasex phenotype variability in males than in females is a fundamental aspect of the gender differences in humans. Dev. Psychobiol. 51, 198-206. doi: 10.1002/dev.20358

Machin, S., and Pekkarinen, T. (2008). Global sex differences in test score variability. Science 322, 1331-1332. doi: 10.1126/science. 1162573

Maylor, E. A., Reimers, S., Choi, J., Collaer, M. L., Peters, M., and Silverman, I. (2007). Gender and sexual orientation differences in cognition across adulthood: age is kinder to women than to men regardless of sexual orientation. Arch. Sex. Behav. 36, 235-249. doi: 10.1007/s10508-006-9155-y

Nakagawa, S., Poulin, R., Mengersen, K., Reinhold, K., Engqvist, L., Lagisz, M., et al. (2015). Meta-analysis of variation: ecological and evolutionary applications and beyond. Methods Ecol. Evol. 6, 143-152. doi: 10.1111/2041-210X.12309

Nowell, A., and Hedges, L. V. (1998). Trends in gender differences in academic achievement from 1960 to 1994: an analysis of differences in mean, variance, and extreme scores. Sex Roles 39, 21-43. doi: 10.1023/A:1018873 615316

Petersen, J. L., and Hyde, J. S. (2010). A meta-analytic review of research on gender differences in sexuality. Psychol. Bull. 136, 21-38. doi: 10.1037/a00 17504

Printzlau, F., Wolstencroft, J., and Skuse, D. H. (2017). Cognitive, behavioral, and neural consequences of sex chromosome aneuploidy. J. Neurosci. Res. 95, 311-319. doi: 10.1002/jnr.23951

Re, A. C. D. (2013). compute.es: Compute Effect Sizes.

Reinhold, K., and Engqvist, L. (2013). The variability is in the sex chromosomes. Evolution 67, 3662-3668. doi: 10.1111/evo.12224
Stoet, G., and Geary, D. C. (2013). Sex differences in mathematics and reading achievement are inversely related: within- and across-nation assessment of 10 years of PISA data. PLoS ONE 8:e57988. doi: 10.1371/journal.pone.0057988

Stoet, G., and Geary, D. C. (2015). Sex differences in academic achievement are not related to political, economic, or social equality. Intelligence 48, 137-151. doi: 10.1016/j.intell.2014.11.006

Stoet, G., and Geary, D. C. (2018). The gender-equality paradox in science, technology, engineering, and mathematics education. Psychol. Sci. 29, 581-593. doi: $10.1177 / 0956797617171974$

Tulving, E. (2002). Episodic memory: from mind to brain. Annu. Rev. Psychol. 53, 1-25. doi: 10.1146/annurev.psych.53.100901.135114

Viechtbauer, W. (2010). Conducting meta-analyses in R with the metafor package. J. Stat. Softw. 36, 1-48. doi: 10.18637/jss.v036.i03

Voyer, D., Voyer, S., and Bryden, M. P. (1995). Magnitude of sex differences in spatial abilities: a meta-analysis and consideration of critical variables. Psychol. Bull. 117, 250-270. doi: 10.1037/0033-2909.117.2.250

Wai, J., Cacchio, M., Putallaz, M., and Makel, M. C. (2010). Sex differences in the right tail of cognitive abilities: a 30year examination. Intelligence 38, 412-423. doi: 10.1016/j.intell.2010.04.006

Wierenga, L. M., Doucet, G. E., Dima, D., Agartz, I., Aghajani, M., Akudjedu, T. N., et al. (2020). Greater male than female variability in regional brain structure across the lifespan. bioRxiv 1-42. doi: 10.1101/2020.02.17.952010

Zechner, U., Wilda, M., Kehrer-Sawatzki, H., Vogel, W., Fundele, R., and Hameister, H. (2001). A high density of $\mathrm{x}$-linked genes for general cognitive ability: a run-away process shaping human evolution? Trends Genet. 17, 697701. doi: 10.1016/S0168-9525(01)02446-5

Conflict of Interest: The authors declare that the research was conducted in the absence of any commercial or financial relationships that could be construed as a potential conflict of interest.

Copyright (C) 2020 Asperholm, van Leuven and Herlitz. This is an open-access article distributed under the terms of the Creative Commons Attribution License (CC BY). The use, distribution or reproduction in other forums is permitted, provided the original author(s) and the copyright owner(s) are credited and that the original publication in this journal is cited, in accordance with accepted academic practice. No use, distribution or reproduction is permitted which does not comply with these terms. 